\title{
The Next Generation of Wireless Communication Using Li-Fi (Light Fidelity) Technology
}

\author{
Yusuf Perwej* \\ Department of Information Technology, Al Baha University, Al Baha, Kingdom of Saudi Arabia (KSA) \\ *Corresponding author: yusufperwej@gmail.com
}

\begin{abstract}
Today scenario almost all the peoples are using internet to fulfill their task through a wired or wireless network. The speed of internet is an important issue and everyone be it business, organizations, entrepreneurs, and institutions is thrust for getting correct information at the correct time and correct place. Then need rapid internet connectivity, technology and huge spectrum of channels. The wireless-fidelity (Wi-Fi) gives you the speed of 150 Mbps, according to standards of 1EEE 802.11n, but not sufficient to fulfill the necessity of required users over the network. In order to remove this deficiency of Wi-Fi, a new technology is developed by German physicist, professor Harald Haas is known as Li-Fi. The Li-Fi is a wireless communication system in which light is used as a carrier signal instead of traditional radio frequency as in Wi-Fi. Li-Fi is a technology that utilizes a light emitting diode to transmit data wirelessly. It uses a bidirectional and the wireless mode of communication using light. In this paper, I am aiming to demonstrate a close-up view about Li-Fi Technology. This technology we can obtain the data rate greatly speedier than $10 \mathrm{Mbps}$, which is a lot greater than our LAN (Local Area Network). Li-Fi is very remunerative over Wi-Fi as it uses VLC by which we can make use of the maximum $60 \mathrm{GHz}$ spectrum. Li-Fi confer transmission of data via LED bulb whose intensity change in an excessively faster speed that it could not be able to be explored by the human eye. Li-Fi is confined to the illuminated area, endue a tremendously controllable environment. The signals cannot travel via walls and are fully secure essentially disassemble the threat of data being hacked remotely.
\end{abstract}

Keywords: Wi-Fi, Li-Fi (Light Fidelity), RF, wireless communication, LED (Light Emitting Diode), photo dectector

Cite This Article: Yusuf Perwej, "The Next Generation of Wireless Communication Using Li-Fi (Light Fidelity) Technology.” Journal of Computer Networks, vol. 4, no. 1 (2017): 20-29. doi: 10.12691/jcn-4-1-3.

\section{Introduction}

In wireless communication network which we use, face the difficulty of access speed when connected to various devices. It creates a general problem when connected to multiple users, the fixed bandwidth which is available for the particular user makes it more difficult to revel high data transfer rates and connect to a secure network. When the many users increases, it creates some aperture [1] between the connections through which it is convenient for the hackers to get through the connections. Nowadays, the rising demand for wireless data communication, the existing radio spectrum below $10 \mathrm{GHz}$ has become inadequate. The wireless communication company has replied to this challenge by considering the radio spectrum beyond $10 \mathrm{GHz}$. There are many reasons which have been considered for the necessity to converge IEEE 802.x wireless network technologies [2]. Expected to ever-increasing stipulation for wireless communications, Wi-Fi is facing many challenges, manageability, service scalability, capacity, namely interoperability, cost effectiveness, availability, efficiency and security. In the line of discovering these issues, the scientist has discover a new substitute method for wireless communication using $\mathrm{Li}-\mathrm{Fi}$ technology, where the data is delivered via visible light alternatively of radio. Li-Fi is principally transmission of data, via the illumination of distinctive LED bulbs and using a photo detector to explore the signals. Li-Fi is the solution as it is inexpensive and much more impressive than Wifi. Li-Fi uses visible light spectrum alternatively of radio waves for relocating of data [3].

The Chair of Mobile Communication at the University of Edinburgh, is recognized as the inventor of Li-Fi. He coined the term $\mathrm{Li}-\mathrm{Fi}$ and is the Co- inventor of pure $\mathrm{Li}-\mathrm{Fi}$ company. He provides a demonstration of a [3] Li-Fi prototype at the TED Global conference in Edinburgh, since $12^{\text {th }}$ July 2011 . He utilization a table lamp with an LED bulb to transfer a video of a blooming flower that was then projected onto a screen. This technology utilizes a part of the electromagnetic spectrum that is still not very much utilized the visible spectrum. Light is actually significantly part of our life of millions and millions of years and does not have any major harmful influence. Additionally, there is 10,000 times more space accessible in this spectrum and just counting on the bulbs in utilizes, it also multiplies to 10,000 times more accessible as an infrastructure, worldwide. Li-Fi is member of optical wireless communication family and wireless communication is one of branches of telecommunications. Be aware the wireless communication, we must first of all to know what 
are telecommunication, communication systems and a variety of networks and then come in a short-range wireless communication which include the Li-Fi. Now we can classify of telecommunication by type channel as shown in Figure 1, the main types of channels are wired telecommunication and wireless telecommunication, wired telecommunication like as cables, coaxial, twisted wire, fiber optics as well as wireless telecommunication such as RF (Radio Frequency) and Optical wireless communication (OWC).

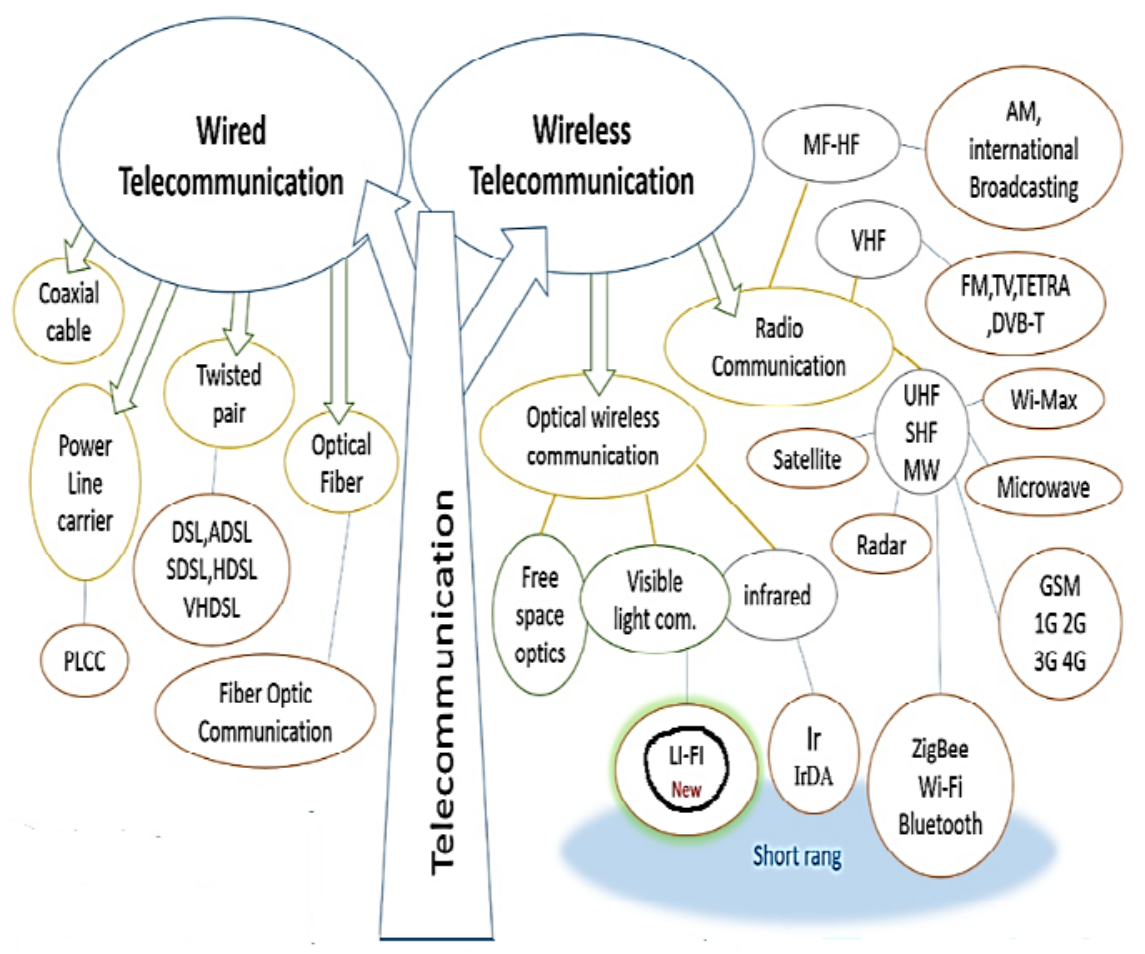

Figure 1. The Classify of Telecommunication

Li-Fi is a VLC, visible light communication, technology invented by a team of scientists including $\mathrm{Dr}$ Gordon Povey, Prof. Harald Haas and Dr Mostafa Afgani at the University of Edinburgh. The term Li-Fi was coined by Prof. Haas when he amazed people by streaming high-definition video from a standard LED lamp [4]. Li-Fi is now part of the Visible Light Communications (VLC) PAN IEEE 802.15.7 standard. Light fidelity technology is a technology that has derived attention in the current years due to its ability to obtain high multiplexing as an outcome of combining thousands of data sources. In this paper respectively investigates and analyzes the Li-Fi (Light Fidelity) Technology.

\section{Issue In Wireless Communication}

The Wi-Fi is a local area wireless computer networking technology that assents electronic devices to network, mainly dealing the 2.5 gigahertz $(12 \mathrm{~cm}) \mathrm{UHF}$ and 5 gigahertz $(6 \mathrm{~cm})$ SHF bands. They are based on the Institute of Electrical and Electronics Engineers (IEEE) 802.11 standards. The computers and handsets enabled with this technology use radio wave to send and receive data somewhere within the range of a base station. The whole stations serving a single radio frequency channel and transmissions on this channel are received by whole stations within range. A general wireless access point using $802.11 \mathrm{~g}$ or $802.11 \mathrm{~b}$ with a stock antenna might have a range of $30 \mathrm{~m}$ indoors and $110 \mathrm{~m}$ outdoors. IEEE $802.11 \mathrm{n}$, [2] in spite of, can more than double the range also varies with frequency band. In wireless communication systems, e.g. 3G, 4G, etc.., a coming trouble is expected due to the shortage of sufficient Radio Frequency (RF) resources. This obstruction in bandwidth can't support the growth in demand for high data rates and the huge numbers of communication systems. Even though, spectrum congestion decreases when we use high frequencies to transfer data, but this not a convenient solution, because this part of the spectrum requires complex equipment and causes high cost systems. Again spectrum assignments and operational restriction are not consistent worldwide. Next the 802.11b and 802.11g use the $2.4 \mathrm{GHz}$ spectrum, which is intensive with other devices. Another limitation power consumption is highly differentiated to other standards, making battery life and heat an anxiety. Actually, there are numbers of technologies that provide practical and applicable solutions to this issue, but in this paper, we will focus on Light Fidelity (Li-Fi) technology [3].

\section{What is Li-Fi?}

$\mathrm{Li}-\mathrm{Fi}$ is a state-of-the-art technology for short range wireless communication system which is [5] convenient for data transmission via LEDs by illumination. Light Fidelity or Li-Fi is a Visible Light Communications (VLC) system running wireless communications travelling at very exalted speeds. The Li-Fi utilization common household LED (light emitting diodes) light bulbs to enable data transfer, boasting speeds of up to 224 gigabits 
per second. The term Li-Fi was coined by University of Edinburgh Professor Harald Haas during a TED Talk in since 2011. Haas speculates light bulbs that could act as wireless routers. The concept of $\mathrm{Li}-\mathrm{Fi}$ is a data communication on fast flickering of light which is not detected by the human eye. However, it is focused on photo detector which transfigure the on-off state into binary digital data. Assume that the LED is on, you transmit a digital 1 , consider it's off you transmit a 0 , they can be switched on and off very fast, which gives benignant favorable moment for transmitting data. It is possible to encode data in the light of differing the rate at which the LEDs flicker on and off to give various strings of $1 \mathrm{~s}$ and 0 s shown in Figure 2. The Li-Fi is designed to use LED light bulbs normally to those at this time use in many energy-conscious homes and offices. In spite of, LiFi bulbs are outfitted with a chip that modulates the light imperceptibly for optical data transmission [4]. Li-Fi data is transmitted by the LED bulbs and accepted by photoreceptors. The Li-Fi technology is being developed into an omnipresent systems technology. They consist of application specific combinations of light transmitters, [5] light receivers including solar cells, efficient computational algorithms and networking potential that can be deployed in an extensive range of communication scenarios and in a diversity of device platforms. Light is beforehand used for data transmission in fiber-optic cables and for point to point links. Nevertheless, Li-Fi is a special and a novel combination of technologies that permits it to be universally adopted for mobile extremely high speed internet communications.

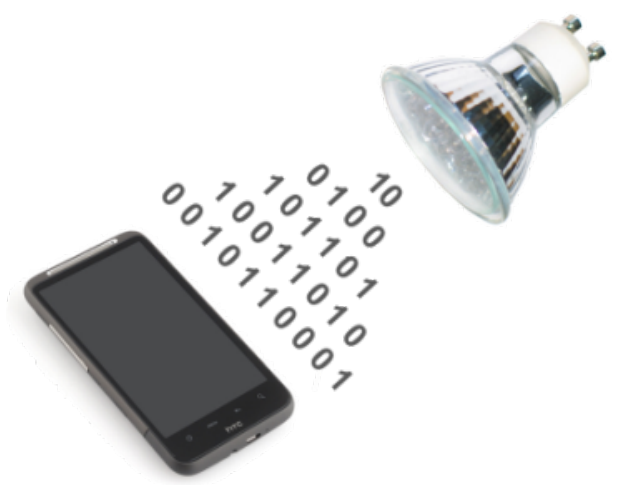

Figure 2. The Data Transmission via LEDs

\section{The Genesis of Li-Fi}

The Professor Harald Haas, from the University of Edinburgh is widely recognized as the main founder of $\mathrm{Li}-\mathrm{Fi}$. He coined the term $\mathrm{Li}-\mathrm{Fi}$ and is chair of mobile communications at the University of Edinburgh and co-founder of the PureLiFi company. The normal term visible light communication (VLC), contain any utilization of the visible light portion of the electromagnetic spectrum to transmit information. The D-Light project at Edinburgh's Institute for Digital Communications was funded from since January 2010 to since January 2012. Haas produced this technique since 2011 TED Global talk and helped start a company to market it. The PureLiFi, formerly PureVLC, is an original equipment manufacturer (OEM) firm sets [6] up to commercialize Li-Fi products for integration with current LED lighting systems. Li-Fi technology is not an entirely new invention. Light signals (infrared) have been used since the 80 s of last century, mostly in TV remote controls. The ultimate success was achieved during 2011, when the first Gigabit-class Li-Fi was developed. Since October 2011, industry groups have formed the Li-Fi alliance, to encourage high-speed optical wireless systems and to conquer the tight amount of radio-based wireless spectrum available by harness a completely different part of the electromagnetic spectrum. Since August 2013, data rates of over $1.6 \mathrm{Gbit} / \mathrm{s}$ were demonstrated over a single color LED. In October 2013, it was announced Chinese manufacturers were working with Li-Fi development kits. Again in April 2014, the Russian company Stins Coman declare the development of a [4] Li-Fi wireless local network called BeamCaster. Their current module transfers data at 1.25 gigabytes per second, but they predict the boosting speeds up to $5 \mathrm{~GB} /$ second in the near future. In 2014 a new record was established by Sisoft (a Mexican company) that was capable of transferring data at speeds of up to $10 \mathrm{Gbit} / \mathrm{s}$ across a light spectrum emitted by LED lamps. Comparable Wi-Fi, Li$\mathrm{Fi}$ is wireless and uses similar 802.11 protocols, but it uses a visible light communication (as an alternative of radio frequency waves), which has much extensive bandwidth.

\section{The Component of Li-Fi System}

In this section we are discussing the various components of Li-Fi system.

\subsection{The LEDs}

The light-emitting diode (LED) is a semiconductor device that emits light when an electric current is passed via it. Light is produced when the particles that carry the current (known as electrons and holes) combine together within the semiconductor material shows in Figure 3. The light is not explicitly bright, but in most [3] LEDs it is monochromatic, occurring at a single wavelength. The light is procreate within the solid semiconductor material, LEDs are mentioned as solid-state devices. The word solid-state lighting, which include organic LEDs distinguishes. These lighting technology from other sources that use heated filaments (incandescent and tungsten halogen lamps) or gas discharge (fluorescent lamps). Preliminary LEDs manufacture only red light, but modern LEDs can manufacture various different colors, including red, green, and blue (RGB) light. Nowadays advances in LED technology have made it possible for LEDs to fabricate white light as well. A nice example of an LED is the led status indicators on your keyboard for NUM Lock, Scroll Lock and Caps Lock.

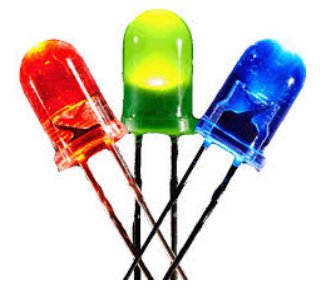

Figure 3. The LEDs 


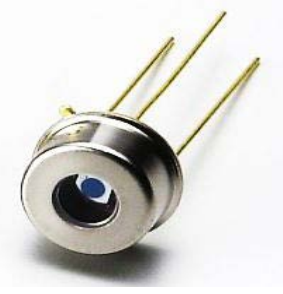

Figure 4. The Photodiode

\subsection{The Photodiode}

A photodiode is a device that helps in the metamorphose of light into electrical current shows in Figure 4. This is made of semi-conductor material and containing a $p-n$ junction, it is designed to function in reverse bias. The current is propagated in the photodiode when photons are absorbed and a very less amount of current is also propagated when there is no existing light. Accompanied by the increase of the surface area, photodiodes have lingering [3] response times. Photodiode technology has been victorious and widely used due to its normal and low-cost rugged structure. Photodiodes have two apart operation modes, firstly namely photovoltaic mode and secondly photoconductive mode. In photovoltaic mode, credence in light is non-linear and the dynamic range achieved is justly small and highest speed is also not acquire in photovoltaic mode. In photoconductive mode, the credence on the light is very linear and opposite voltage has no significant impact on light, but has a weak impact on dark current (current achieved without light). The Photodiodes is comprehensively used in the electronics industry, especially in detectors and wide bandwidth optical telecommunications systems.

\subsection{The Image Sensor}

An image sensor is an electronic, photosensitive device which transforms an optical image into an electronic signal. They are composed of millions of photodiodes and is used as an image receiver in digital imaging equipment. An image sensor is competent of reacting to the impact of photons, thus converting them into an electrical current that is then passed onto an analog-digital converter is shown in Figure 5. The most usual types of image sensors are CCD and CMOS sensors. Image sensors are commonly used in camera modules and other imaging devices. At this time, the utmost common image sensors are digital charge-coupled device (CCD) or complementary metal oxide semiconductor (CMOS) active pixel sensors. In a camera, a photo electronic image sensor transforms the light passing through the lens into per-photodiode charges of varying sizes.

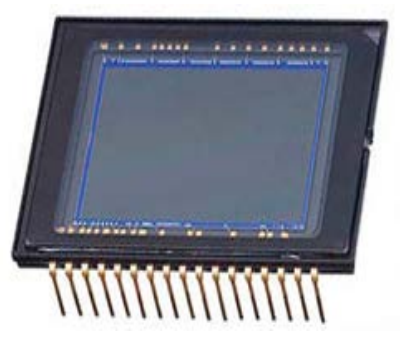

Figure 5. The Image Sensor

\section{The Working Principle of Li-Fi}

The Li-Fi and Wi-Fi are quite similar as both transmitting data electromagnetically. The functioning of new Li-Fi technology is just easy. You will have a light source at one end like an LED and a photo detector (Light Sensor) on the other end. Although, Wi-Fi uses radio waves while $\mathrm{Li}-\mathrm{Fi}$ runs on visible light. The $\mathrm{Li}-\mathrm{Fi}$ technology is implemented using white LED light bulbs used for [3] illumination by applying a perpetual current. However, by fast variations of the current, the light output can be made to vary at very high speeds. We essential at all for sending data are some LEDs and a controller that cods data into those LEDs and for receiving data, we essential an Image Sensor, Photodiode which is used as a detector. The binary data are seizing by few light receptors are required, and are installed on all types of connected devices, from computers to tablets, to phones, televisions or appliances shows in Figure 6. The matter specialist makes clear that the light pulses are imperceptible to [5] the human eye, without causing harm or discomfort of any kind. Therewith, any lamp or flashlight can become a hotspot. How Li- Fi works are quite simple because you have a light on one end (an LED), and a photodetector (light sensor) on the other. If the LED is ON, the photo detector registers a binary one; else it's a binary zero. Flash the LED sufficient times and you build up a message. Using an array of LEDs [7], and probably a few different colors, and very shortly you are dealing with data rates in the range of hundreds or megabits per second, this is endowed by the flickering of LED light bulbs to create binary code (on $=1$, off $=0$ ), and is done at surpassing rates than the human eye can detect. The excess LEDs in your lamp, the excess data it can process.

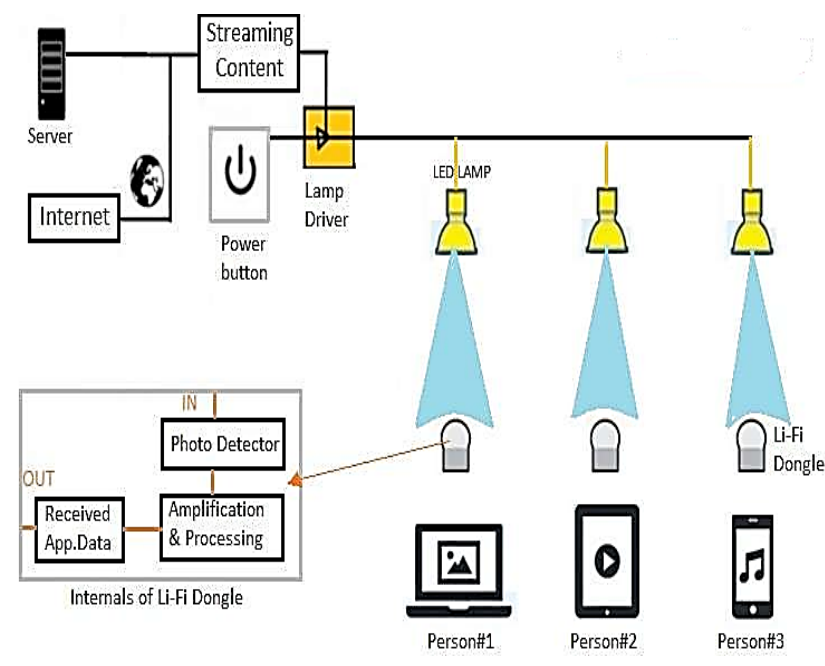

Figure 6. The Working Of Li-Fi Communication

If LED shifting data at a sluggish rate, so millions of LEDs with one micron size are installed in the bulb. The reduction of size of LEDs does not shortage, its ability to shift data or intensity on the opposite it increases the efficiency of one light bulb to transmit the data at a suddenly higher rate. The Figure 7 shows a brief connection of internet [8] with LED and information retrieved on the Tab. Moreover, these micro-LEDs are ultimately just pixels and at one micron, these LEDs would be a lot lesser than those in your Smartphone's 
retina display. You could have a large array of these LEDs that double up as a room's light source and a display and provides networking ability on the side. The model of Li-Fi LED light shows in Figure 8.
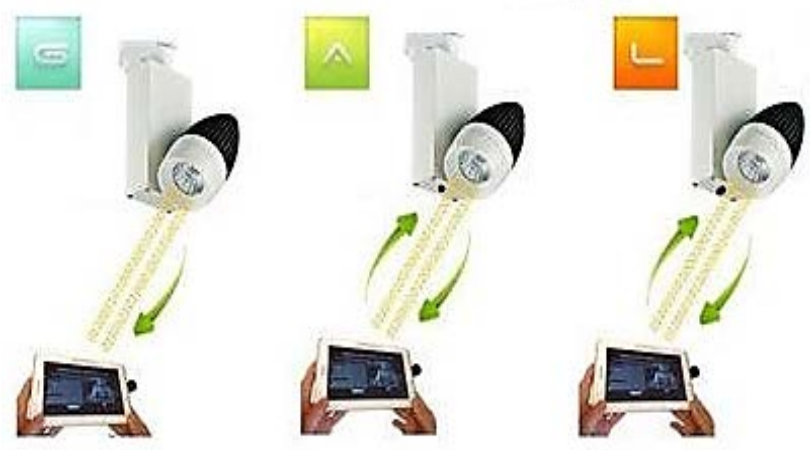

Figure 7. The Connection Between LED And Tab

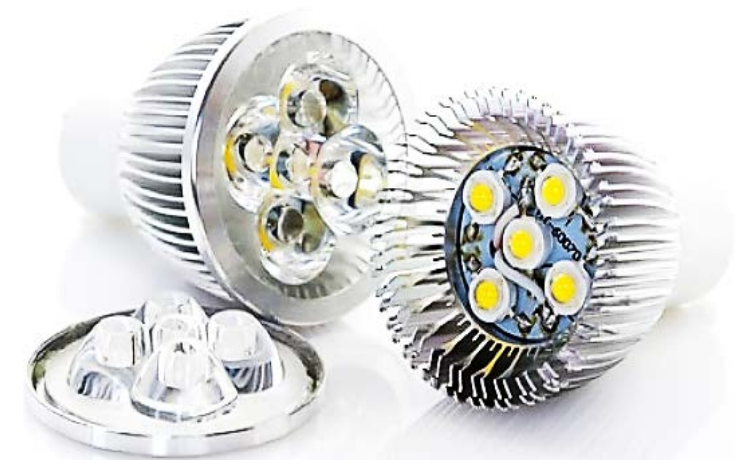

Figure 8. The Model Of Li-Fi LED Lights

\section{The Tx and Rx in Li-Fi}

Let's start with TX and RX in other words Transmit and Receive. Adding the $\mathrm{x}$ is a shorthand. An $\mathrm{x}$ implies something crossing as in Railway Xing (crossing). The data are crossing or traveling over a cable or via a signal in the air. Li-Fi is transmission of data via illumination of the LED by taking the fiber out of the fiber optics by sending data via the LED light bulb that varies in intensity intense than the human eye can follow. If the LED [7] is ON, the user can transmit a digital string of 1, if it's OFF then the user can transmit a string of 0 . It can be switched ON and OFF very speedily, which gives instant favorable moment for transmitting data. It is believable to encode data in the light by varying the rate at which the LEDs flicker $\mathrm{ON}$ and OFF to move on dissimilar strings of $1 \mathrm{~s}$ and 0s. Actually, this technology transfers thousands of streams of data at the same instant in parallel in higher speed with the help of the special modulation technique using a unique signal processing technology. The light used to transmit the data is called D-light by Herald Hass, the originator of Li-Fi [4]. The Tx side will transmit the data. It is connected to arrays of LEDs via which data is transferred. This data will be received by the receiving side $(\mathrm{Rx})$ side shows in Figure 9. The receiver side will receive the data that is transmitted via the led panel. These LEDs can be displayed to the Hyper Terminal on the PC by connecting a serial uart.
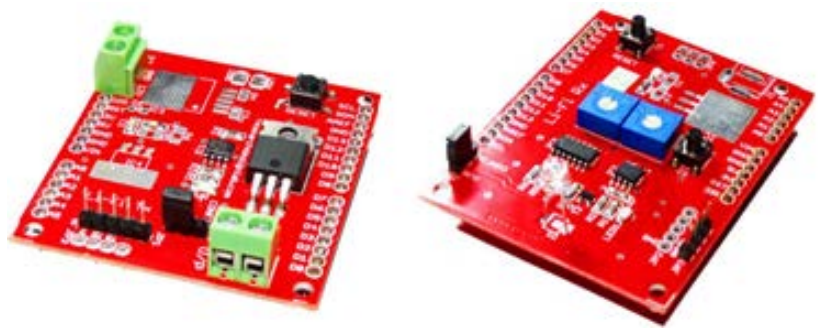

Figure 9. The Li-Fi Tx And Rx

\section{The Li-Fi Communication}

This section deals with the physical layer, modulation formats and frequency spectrum that are associated with Li-Fi communication. The IEEE 802.15.7 [2] standard defines the physical layer (PHY) and media access control (MAC) layer. The standard is capable of delivering enough data rates to transmit audio, video and multimedia services. It takes into account the optical transmission mobility, its similarity with artificial lighting present in infrastructures, the deviance which may be caused by interference originate by the comprehensive lighting. The MAC layer allows using the link with the other layers like the TCP/IP protocol. The standard defines three PHY layers with dissimilar rates.

- The PHY I was established for outdoor application and serve from $11.67 \mathrm{kbit} / \mathrm{s}$ to $267.6 \mathrm{kbit} / \mathrm{s}$.

- The PHY II layer permission to reach data rates from 1.25 Mbit/s to $96 \mathrm{Mbit} / \mathrm{s}$.

- The PHY III is used for many emissions sources with a distinctive modulation method called color shift keying (CSK). PHY III can convey rates from $13 \mathrm{Mbit} / \mathrm{s}$ to $98 \mathrm{Mbit} / \mathrm{s}$.

The modulation formats valid for PHY I and PHY II are the coding, on-off keying (OOK) and variable pulse position modulation (VPPM). The Manchester coding applied to the PHY I and PHY II layers include the clock inside the transmitted data by representing a [8] logic 0 with an OOK symbol "01" and a logic 1 with an OOK symbol " 10 ", all with a DC component. The DC component averts the light extinction in case of an extended line of logic 0 . The Optical Orthogonal Frequency Division Multiplexing (O-OFDM) modulation methods which have been improved for data rates, multiple-access and energy efficiency which can be utilized for Li-Fi communication. The novel high-speed optical wireless models utilize in both indoors and outdoors. The Li-Fi confer [7] resources for ODM (Original Design Manufacturers) and OEM (Original Equipment Manufacturer) developers to create exciting new products. Accompanied the emergence of high-speed cable connections like Thunderbolt and USB 3.0, the stage is set for a wireless equivalent. When $\mathrm{Wi}-\mathrm{Fi}$ is very famous for pervasive $100+$ Mbps service, multi-Gigabit short-range optical wireless interconnects provide a substitute to the proposed [9] Gigabit RF solution. For communication aim light wave carries data in a rapid manner, but in radio wave the data transfer rate is sluggish. Therefore that cause we are looking for light wave communication. It is shown by the Figure 10. However, 
the drawback that light does not penetrate through the walls and no communication in darkness, so if one moves from one room to another he will require a wired bulb in that room too.

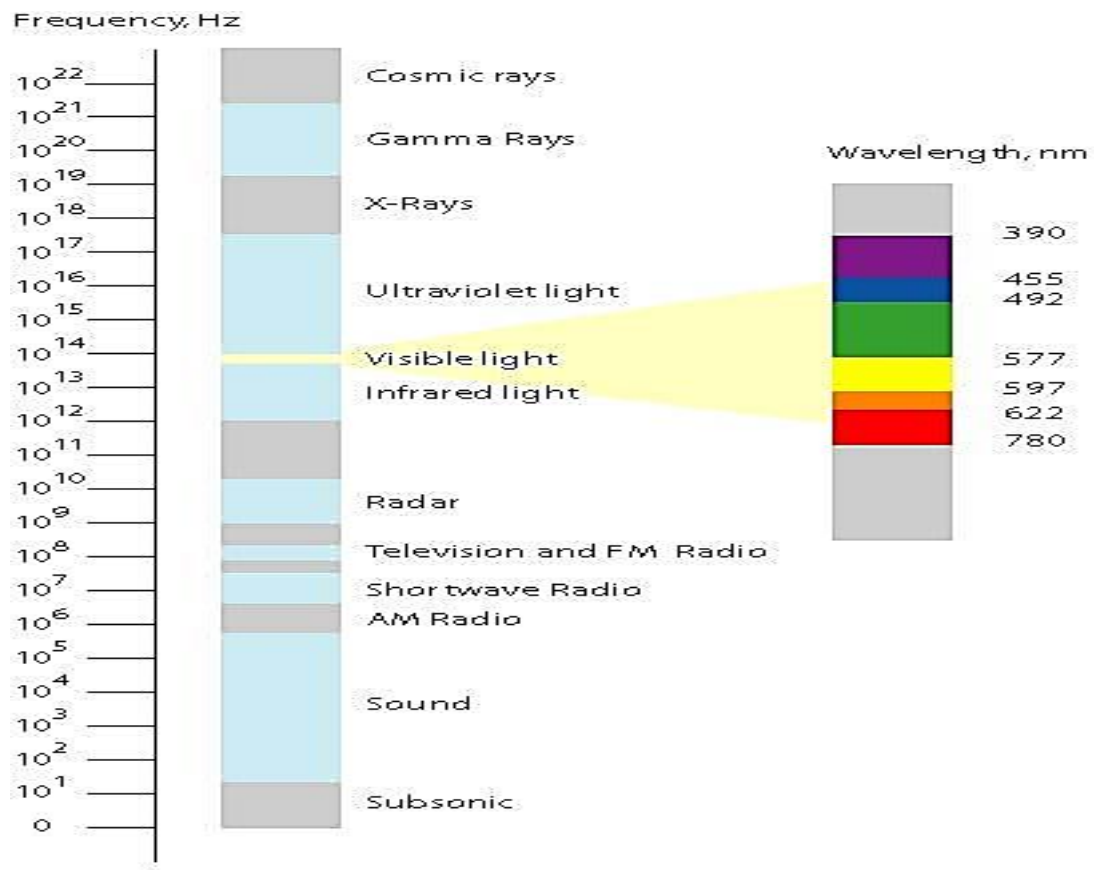

Figure 10. The Radio Wave And Light Wave Frequency Spectrum

\section{The Modulation and Li-Fi System}

The Li-Fi is based on visible light communication (VLC) and used optical wireless communication (OWC) technique this technique you can alter by OWC technique VLC modulation to Li-Fi modulation such as SIM OFDM modulation or OFDMA (OFDM multi-access) for many users. The VLC can only be perceived as an IM/DD system, which means that the modulation signal has to be both pure valued and unipolar. The application of [10] the well-researched and developed modulation schemes from the field of RF communications. This technique like as on-off keying (OOK), pulse-position modulation (PPM), pulse-width modulation (PWM) and unipolar M-ary pulse amplitude modulation (M-PAM) can be applied in a relatively uncomplicated fashion, a more resilient technique such as OFDM is required. The OFDM permits an adaptive bit and energy loading of various frequency sub-bands in pursuance of to the communication channel In order to actually send out data [11] via LED, like any multimedia data, it is essential to modulate these into a carrier signal. This carrier signal consists of light pulses sent out at less intervals. The system used in optical wireless communication (OWC) technique is Optical Orthogonal Frequency Division Multiplexing (O-OFDM).

In fact send out data through LED, like any multimedia data, it is necessary to [12] modulate these into a carrier signal. This carrier signal comprises of light pulses sent out at short intervals. Fundamentally depends on the selectivity modulation scheme. Two of which are firstly Subcarrier pulse (SCP) is presented which is beforehand established as VLC-standard by the VLCC and secondly modulation scheme to be addressed is called frequency shift keying (FSK).

- Pulse-position modulation (PPM): Sub-Carrier Inverse PPM (SCIPPM), a method whose structure is divided into two parts firstly sub-carrier part and secondly DC part. The DC part is only for lighting or pointing. When there is no necessity of lighting or indicating SCPPM (Sub-Carrier PPM) is used for VLC to save energy.

- Frequency Shift Keying (FSK): In frequency shift keying (FSK) data is represented by change frequencies of the carrier wave. Prior to transmitting two distinct values ( 0 and 1 ), there necessity to be two distinct frequencies. This is also the normal form of frequency shift keying, called binary frequency shift keying (BFSK).

- SIM-OFDM Technique (Sub-Carrier Index Modulation OFDM): Distinct conventional OFDM illustrate in the SIM-OFDM technique partitioned the serial bit stream B into two bits-sub streams of the same length [13]. The next process is to select two apart modulation alphabets MH and ML (i.e. 4QAM and BPSK) to be allocated to the first and the second subsection of the first bit-sub stream.

\section{The Viewpoint of Li-Fi}

The Li-Fi technology can be used for different purposes, it matters the data transmission via LEDs thus all the screens which illuminate light can be served as a platform for data communication. The Li-Fi cloud is a software solution enabling the user to control all the characteristics within a data centric Li--Fi environment. In Figure 11 shows how the Li-Fi cloud will get communicated with other electronic devices. The Li-Fi using visible light as an alternative of gigahertz radio waves. At this time there are 1.4 billion base stations which consume more energy and its competence is less than five percent and we have a total of almost five million mobile phones which transfer more than 600 terabytes [6] of data every month which 
showcase the reality that wireless has become usefulness. The screen of the mobile phone, television, bulbs can gesture as a source of light. On the contrary, the receiving platform, the photo detector can be replaced by a camera in mobile phone for scanning and access data. So many applications are Li-Fi for desktops, hospitals, Li-Fi in cities, smart guides, museums, smart card, Li-Fi for schools, hotels, fairgrounds, events, indoor, [14] access control and identification crisis, malls, airport and dangerous environments like thermal power plants. The forerunner of data transmission through the blinking of the LEDs can create wireless internet access with data transmission speeds of close to 10Gbit/s, theoretically, permit a high-definition film to be downloaded in 35 seconds which is 250 times faster than excellent broadband. These advantages come at a quintuple transits currently offering fiber optic lines, [15] to advantage from this technology requires a luminous router which is competent of emitting the binary signal.

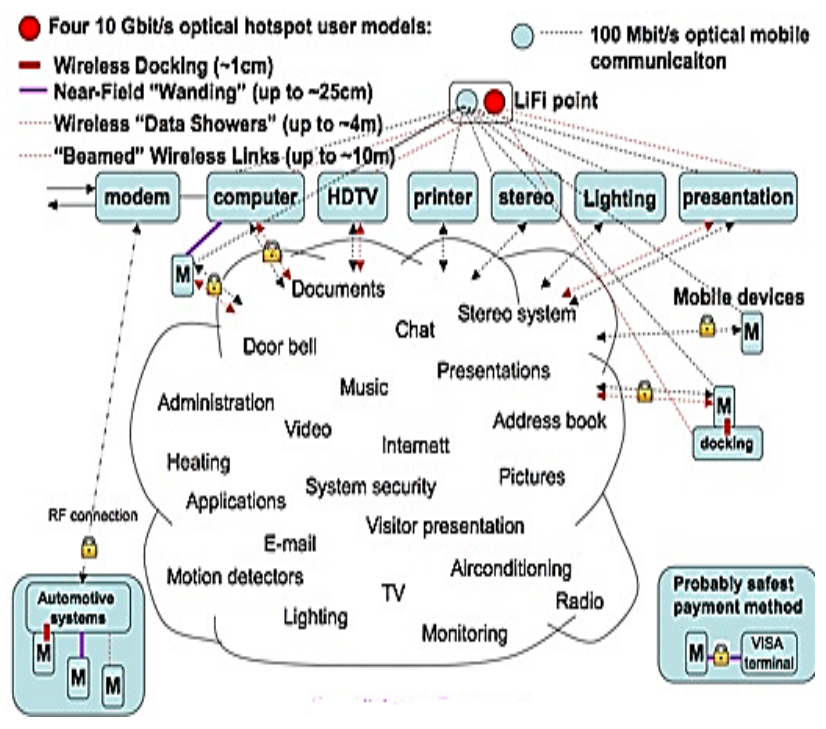

Figure 11. The Li-Fi Communication With Other Electronic Devices

\section{Benefits of Li-Fi}

The advantage of Li-Fi emerge as a result of unwind the large amount of license-free and unbroken electromagnetic spectrum that falsehood in the infrared and visible light regions. The main advantage are Li-Fi utilization LEDs and visible light to transmit data wirelessly as a substitute for short-range radio. It does not require any special LED bulbs, however, only standard LED bulbs which are used in houses and offices. Li-Fi is a bidirectional technology, nowadays LEDs are used only to transmit data due to feasibility and cost issues involved. Li-Fi utilization visible light band, between 400 and $800 \mathrm{THz}$ of the electromagnetic spectrum to transmit data. The biggest benefit of this technology is speed [3] blazing rapidly data transmission speeds up to 100 times that of Wi-Fi. It has beforehand hit speeds of 224Gbps in the laboratory, so long as the tests under real-life conditions have reached up to $1 \mathrm{Gbps}$, a leap from average Wi-Fi speeds of between $300 \mathrm{Mbps}$ and $720 \mathrm{Mbps}$. Li-Fi could also discover widespread adoption in aircraft, as Li-Fi signals do not interfere with radio waves that pilots utilization to communicate. The computers or mobile devices will also necessitate to be fitted with sensors that can read the light signals and convert them into data. The sensors are currently about the size of a smartphone and thus not very suitable for mobile users. Wherever possible there is a light source, there can be Internet. Light bulbs are nowadays ubiquitously in offices, shops, malls, homes and even planes, meaning that high-speed data transmission could be [4] available ubiquitously. Another main superiority of Li-Fi is security. The light cannot pass via inconspicuous structures, Li-Fi Internet is accessible only to the users inside a room and cannot be breached by users in other rooms or buildings. Every visible light information source can be unambiguously identified, so the location of any VLC device can be identified speedily and accurately [16]. The mobile phones and Wi-Fi are unwelcome in certain parts of hospitals, especially around MRI scanners and in operating theatres. Today, many cars previously LED lamps. Traffic signage, traffic lights, and street lamps are taken on the LED technology so there are enormous applications favorable time here.

\section{Application of Li-Fi Technology}

Li-Fi technology can find application in an extensive variety of fields. Li-Fi is particularly appropriate for many popular internet content consumption applications such as video and audio downloads, live streaming. These applications place bulky demands on the downlink bandwidth, but need minimal uplink capacity [2,4]. A detailed discussion of its various applications is given below.

- Smart Lighting: The private or public lighting including street lamps can be utilized to endow LiFi hotspots and the same communications and sensor infrastructure can be consumed to monitor and control lighting and data.

- Mobile Connectivity: The smart phones, tablets, laptops, and other mobile devices can interconnect directly using Li-Fi. Use short range links give very high data rates and also provides security.

- RF Avoidance: Some people's insistence they are hypersensitive to radio frequencies and are looking for an alternative. $\mathrm{Li}-\mathrm{Fi}$ is a better solution to this problem.

- Location Based Services (LBS): The extremely accurate location-specific information services such as advertising and navigation that enables the recipient to receive appropriate, [17] pertinent information in a timely manner and location.

- RF Spectrum Relief: Additional capacity demands of cellular networks can be offloaded to Li-Fi networks where available. This is circumstantially effective on the downlink where bottlenecks tend to occur.

- Education: Li-Fi is the leading technology that makes better the internet accessibility speed with high bandwidth. Consequently, educational institutions and organizations can use this technology for internet access with fast speed for video conference, digital tutorial downloads and online learning.

- Disaster Management: Li-Fi can be installed, the communication in natural disaster times such as earthquakes, cyclones, tsunami or hurricanes. Li-Fi 
bulbs could be fixed in the streets to provide light and economical high speed internet access in each corner of the street.

- Hazardous Environments: Li-Fi provides a safe substitute to electromagnetic interference from radio frequency communications in environments like as mines and petrochemical plants.

- Hospital \& Healthcare: Li-Fi no release electromagnetic interference and so does not interfere with medical instruments, nor is it interfered with by MRI scanners.

- Aviation: Li-Fi can be used to reduce weight and cabling and add pliability to seating layouts in aircraft passenger cabins [7] where LED lights are beforehand deployed. In the flight entertainment (IFE) systems can also be supported and integrated with passengers' own mobile devices.

- Game Consoles: A modern idea would be to put sensors on a television in order to receive information from game consoles [17]. This would allow the unit to be placed literally somewhere within the room as long as there is a direct line of sight to the sensor.

- Underwater Communications: Due to strong signal absorption in water, RF use are unsuitable. Acoustic waves have the mostly low bandwidth and disarrange marine life. $\mathrm{Li}$-Fi provides a solution for short-range communications.

\section{The Comparison of Li-Fi \& Wi-Fi \& WiMAX and Bluetooth}

The Table 1 below contains the current wireless technologies that can be used for transferring data between devices today scenario $[18,19]$. This section we are describing the basic difference between $\mathrm{Li}-\mathrm{Fi}$, Wi-Fi, WiMAX, and Bluetooth technologies [20,21].

Table1. The Comparison of Li-Fi \& Wi-Fi \& WiMAX and Bluetooth

\begin{tabular}{|c|c|c|c|c|}
\hline Features & $\mathrm{Li}-\mathrm{Fi}$ & Wi-Fi & WiMAX & Bluetooth \\
\hline Full form & Li-Fi (Light Fidelity) & Wi-Fi (Wireless Fidelity) & $\begin{array}{l}\text { WiMAX (Worldwide } \\
\text { Interoperability for Microwave } \\
\text { Access) }\end{array}$ & $\begin{array}{l}\text { Bluetooth Full From the epithet of } \\
\text { the tenth-century king Harald } \\
\text { "Bluetooth" Gormsson. }\end{array}$ \\
\hline Operation & $\begin{array}{l}\text { Li-Fi transmits data using } \\
\text { light with the help of LED } \\
\text { bulbs. }\end{array}$ & $\begin{array}{l}\text { WiFi transmits data using } \\
\text { radio waves with the help of } \\
\text { Wi-Fi router. }\end{array}$ & $\begin{array}{l}\text { Broadband Wireless } \\
\text { Access. }\end{array}$ & $\begin{array}{l}\text { Anywhere at least two Bluetooth } \\
\text { devices exist. }\end{array}$ \\
\hline Interference & $\begin{array}{l}\text { Do not have any } \\
\text { interference issues similar } \\
\text { to radio frequency waves. }\end{array}$ & $\begin{array}{l}\text { Will have interference issues } \\
\text { from nearby access points } \\
\text { (routers). }\end{array}$ & $\begin{array}{l}\text { WiMAX communications pose a } \\
\text { significant interference threat to } \\
\text { satellite signals transmitted in the } \\
\text { C-band frequency. }\end{array}$ & $\begin{array}{l}\text { Bluetooth devices interfere with } \\
\text { other technologies. }\end{array}$ \\
\hline Technology & $\begin{array}{l}\text { Present IrDA compliant } \\
\text { devices }\end{array}$ & $\begin{array}{l}\text { WLAN802.11a/b/g/n/ac/ad } \\
\text { standard compliant devices }\end{array}$ & $\begin{array}{l}\text { Wireless metropolitan } \\
\text { area network (WMAN) }\end{array}$ & WPAN \\
\hline Applications & $\begin{array}{l}\text { Used in airlines, undersea } \\
\text { explorations, operation } \\
\text { theaters in the hospitals, } \\
\text { office and home premises } \\
\text { for data transfer and } \\
\text { internet browsing } \\
\end{array}$ & $\begin{array}{l}\text { Used for internet browsing } \\
\text { with the help of wifi kiosks } \\
\text { or Wi-Fi hotspots }\end{array}$ & $\begin{array}{l}\text { WiMAX serves a larger } \\
\text { interoperable network. }\end{array}$ & $\begin{array}{l}\text { Bluetooth applications is huge, } \\
\text { because we transact business and } \\
\text { communicate more with people who } \\
\text { are close by than with those who are } \\
\text { far away. }\end{array}$ \\
\hline Merits & $\begin{array}{l}\text { Interference is less, can } \\
\text { pass through salty sea } \\
\text { water, works in a densy } \\
\text { region. }\end{array}$ & $\begin{array}{l}\text { Interference is more, cannot } \\
\text { pass through sea water, } \\
\text { works in less densy region. }\end{array}$ & $\begin{array}{l}\text { WiMAX can be used for long } \\
\text { ranges. It provides broadband } \\
\text { connectivity up to varied ranges, } \\
\text { around } 30 \mathrm{~km} \text {. }\end{array}$ & $\begin{array}{l}\text { Setting up a Bluetooth connection } \\
\text { between two devices is quick and } \\
\text { easy. A Bluetooth headset is } \\
\text { compatible with any other device } \\
\text { that supports Bluetooth, }\end{array}$ \\
\hline Privacy & $\begin{array}{l}\text { In } \mathrm{Li}-\mathrm{Fi} \text {, light is blocked } \\
\text { by the walls and hence } \\
\text { will provide more secure } \\
\text { data transfer }\end{array}$ & $\begin{array}{l}\text { In WiFi, RF signal can not } \\
\text { be blocked by the walls and } \\
\text { hence need to employ } \\
\text { techniques to achieve secure } \\
\text { data transfer. }\end{array}$ & $\begin{array}{l}\text { WiMAX uses X.509 or PKMv2 } \\
\text { as authentication algorithms. } \\
\text { Mandatory-3DES Optional- AES }\end{array}$ & $\begin{array}{l}\text { Bluetooth offers several security } \\
\text { modes, and device manufacturers } \\
\text { determine which mode to include in } \\
\text { a Bluetooth-enabled gadget. }\end{array}$ \\
\hline $\begin{array}{l}\text { Data transfer } \\
\text { speed }\end{array}$ & About 1 Gbps & $\begin{array}{l}\text { WLAN-11n offers 150Mbps, } \\
\text { About 1-2 Gbps can be } \\
\text { achieved using WiGig/Giga- } \\
\text { IR }\end{array}$ & $\begin{array}{l}\text { Works at } 5 \mathrm{bps} / \mathrm{Hz} \text { and can peak } \\
\text { up } \\
\text { to } 100 \mathrm{Mbps} \text { in a } 20 \mathrm{MHz} \\
\text { channel. }\end{array}$ & 800KBps \\
\hline $\begin{array}{l}\text { Frequency of } \\
\text { operation }\end{array}$ & $\begin{array}{l}10 \text { thousand times } \\
\text { frequency spectrum of the } \\
\text { radio }\end{array}$ & 2.4GHz, $4.9 \mathrm{GHz}$ and $5 \mathrm{GHz}$ & $\begin{array}{l}\text { Licensed/Unlicensed } \\
2 \mathrm{G} \text { to } 11 \mathrm{GHz}\end{array}$ & $2.4 \mathrm{GHz}$ \\
\hline Data density & $\begin{array}{l}\text { Works in high dense } \\
\text { environment }\end{array}$ & $\begin{array}{l}\text { Works in less dense } \\
\text { environment due to } \\
\text { interference related issues }\end{array}$ & $\begin{array}{l}\text { Works in high dense } \\
\text { environment }\end{array}$ & Less dense \\
\hline Coverage distance & About 10 meters & $\begin{array}{l}\text { About } 32 \text { meters (WLAN } \\
802.11 \mathrm{~b} / 11 \mathrm{~g}) \text {, vary based on } \\
\text { transmit power and antenna } \\
\text { type }\end{array}$ & Up to 40 miles & About 10 meters \\
\hline $\begin{array}{l}\text { System } \\
\text { components }\end{array}$ & $\begin{array}{l}\text { Lamp driver, LED bulb } \\
\text { (lamp) and photo detector } \\
\text { will make up complete Li- } \\
\text { Fi system. }\end{array}$ & $\begin{array}{l}\text { Requires routers to be } \\
\text { installed, subscriber devices } \\
\text { (laptops, PDAs, desktops) } \\
\text { are referred as stations }\end{array}$ & $\begin{array}{l}\text { There are three main components } \\
\text { of WiMax network architecture. } \\
\text { He first component is the mobile } \\
\text { stations, second network is an } \\
\text { access service network and third } \\
\text { component is connectivity } \\
\text { service network which is } \\
\text { responsible for providing IP } \\
\text { functions. }\end{array}$ & $\begin{array}{l}\text { Four major components: } \\
\text { Radio Unit(radio transceiver) } \\
\text { Baseband Unit (flash memory \& } \\
\text { CPU) } \\
\text { Software Stack (driver software) } \\
\text { Application Software (user } \\
\text { interface) }\end{array}$ \\
\hline
\end{tabular}




\begin{tabular}{|l|l|l|l|l|}
\hline Features & Li-Fi & Wi-Fi & WiMAX & Bluetooth \\
\hline $\begin{array}{l}\text { Power } \\
\text { consumption }\end{array}$ & Medium & Medium & High & Low \\
\hline Cost price & Low & Medium & Medium & Low \\
\hline Working Concept & $\begin{array}{l}\text { Direct Binary Data } \\
\text { Serving }\end{array}$ & Various Topologies & Request/Grant & Master- Slave \\
\hline
\end{tabular}

\section{Challenges for $\mathrm{Li}-\mathrm{Fi}$}

The metamorphose of existing devices into Li-Fi enabled ones. Need of line of sight for communication and it also faces interference from external light sources like [4] the sun. Exalted installation costs until it becomes more commercial. The Internet cannot be utilized without a light source. This could limit the locations and circumstances in [22] which Li-Fi could be used. In view of the fact that it uses visible light, and light cannot penetrate walls, the signal's range is limited by physical barriers. Other sources of light may interfere with the signal. One of the biggest believable drawbacks is the interception of signals outdoors. Sunlight will interfere the signals, outcome in interrupted Internet. An entire new infrastructure for Li-Fi would need to be constructed. Though it draws low power, in order to avail Li-Fi internet services, lights need to be kept ON throughout the day and night. As internet is a requirement of the hour, [23] this will worthless energy more [3] than any other internet [7] system. If you cannot watch games and videos on the internet in the dark during night before sleeping on the bed. When the transceivers are equipped with wide beam, the copies of the same signal from different paths reach the [24] destination with different amount of delay, therefore each path has different length from source to destination. This creates a difficulty called multipath distortion which can reason inter symbol interference that unmercifully degrades the performance.

\section{Conclusion}

The communication, the extremely emerging part in everybody's life is through exchanging information either on wired or wireless devices. In pursuance of to the changing scenario of wireless communication as the electromagnetic spectrum decrease continuously the Li-Fi system will go to provide a greener, safer, optimal and healthier ensuing for communication system. Li-Fi has a thousand times greater swiftness than Wi-Fi. It's provides security as the visible light is unable to infiltrate through the walls, which offer a new era of wireless communication. The existence of semiconductors, the LEDs allow for their electrical current to be dipped and dimmed at immensely high speeds. What's more, the human eye can't even notice the disparity. Comprehensibly, this is incredibly important when use for data transfer where data fed into the LED bulb via signal processing can then be embedded into the light beam at fast speeds to the receiving photodetector (photodiode). These nano scale changes in the rapidly dimming of the bulb can then be transformation of the receiver into a useful digital signal. In this paper, aimed to demonstrate a close-up view about $\mathrm{Li}-\mathrm{Fi}$, including a component of Li-Fi system, Genesis of $\mathrm{Li}-\mathrm{Fi}$, working principle of $\mathrm{Li}-\mathrm{Fi}$, we also brief description about the benefits and application of Li-Fi technology and comparison of various techniques. Finally, the Li-Fi concept is captivated a great deal of interest, because it provides an authentic and very dexterous substitute to wireless device which used radio spectrum. It is superior and implementable in different fields that can't be done with the Wi-Fi and other technologies.

\section{References}

[1] Behrouz A. Forouzan, Data Communications and Networking, Fourth Edition. The McGraw-Hill Companies, Inc, 2007.

[2] IEEE Std. 802.15.7-2011, 2011, IEEE Standard for Local and Metropolitan Area Network, Part 15.7: Short-Range Wireless Optical Communication Using Visible Light, IEEE Std, 2011.

[3] S. Dimitrov and H. Haas, Principles of LED Light Communications: Towards Networked Li-Fi, 2015, Cambridge Univ. Press.

[4] H. Haas, "Wireless data from every light bulb", TED Website [Online]. 2011 Available: http://bit.ly/tedvlc.

[5] Khalid, A. M., Cossu, G., Corsini, R., Choudhury, P., and Ciaramella, E., "1-Gb/s Transmission Over a Phosphorescent White LED by Using Rate-Adaptive Discrete Multitone Modulation,” IEEE Photonics Journal 4, 1465-1473 (Oct. 2012).

[6] H. Haas and C. Chen "What is LiFi" in Proc, 41st European conference on Opt Communication, Valencia, Spain, Sept, 27, 2015, pp: 1-3.

[7] Dobroslav Tsonev, Stefan Videv, Harald Haas, Light fidelity (Li-Fi): towards all-optical networking in Proc. SPIE 9007, Broadband Access Communication Technologies VIII, 900702 (December 18, 2013).

[8] Z. Chen, D. Tsonev and H. Haas, "A novel double-source cell configuration for indoor optical attocell networks", Global Telecommunication Conf. Austin, TX, USA, Dec.8-12, 2014.

[9] Y. Saito, Y. Kishiyama, A. Benjebbour, T. Nakamura, A. Li and K. Higuchi, "Non-orthogonal multiple access (NOMA) for cellular future radio access", Proc. IEEE Veh. Technol. Conf., pp. 1-5, 2013.

[10] S. Randel, F. Breyer, S. C. J. Lee, et al., “Advanced modulation schemes for short-range optical communications,”IEEE Journal of Selected Topics in Quan-tum Electronics, vol. PP, no. 99, pp. 1-10, 2010.

[11] S. Dissanayake and J. Armstrong,"Comparison of ACO-OFDM, DCO-OFDM and ADO-OFDM in IM/DD systems,”Journal of Lightwave Technology, vol. 31, no. 7, pp. 1063-1072, Apr. 2013.

[12] C. Wu, H. Zhang, and W. Xu,“On visible light communication using led array with DFT-spread OFDM,” in IEEE International Conference on Communica- tions (ICC), Sydney, Australia, Jun. 2014, pp. 3325- 3330.

[13] W. Huang, C. Gong, and Z. Xu, "System and waveform design for wavelet pack - et division multiplexing - based visible light communications,”Journal of Light-wave Technology, vol. 33, no. 14, pp. 3041- 3051, Jul. 2015.

[14] S. Dimitrov and H. Haas, Principles of LED Light Communications: Towards Networked Li-Fi. Cambridge, U.K.: Cambridge Univ. Press, Mar. 2015.

[15] A. Farid and S. Hranilovic, "Capacity bounds for wireless optical intensity channels with Gaussian noise,” IEEE Trans. Inf. Theory, vol. 56, no. 12, pp. 6066-6077, Dec. 2010.

[16] Gomez A. , Kai Shi, Quintana C. , Sato M, Faulkner, Thomsen B.C., O’ Brien D, “Beyond 100-Gb/s Indoor Wide Field-of-View Optical Wireless Communications,” Photonics Technology Letters, IEEE , Volume 27, Issue 4 , pp. 367-370, February 2015.

[17] B. Rofoee, K. Katsalis, Y. Yan, Y. Shu, T. Korakis, L. Tassiulas, A. Tzanakaki, G. Zervas, and D. Simeonidou, "First demonstration of service-differentiated converged optical sub-Wavelength and LTE/ WiFiNetworks over GEAN," in Proc. Opt. Fiber Commun. Conf.Exhib., Mar. 2015, pp. 1-3. 
[18] Laudin Molina, Tanguy Kerdoncuff, Dareen Shehadeh ,"WMSP: Bringing the Wisdom of the Crowd to WiFi Networks", IEEE Transactions on Mobile Computing ,Volume: PP, Issue 99, 14 April 2017.

[19] Ioannis Papapanagiotou et. al. "A Survey on Next Generation Mobile WiMAX Networks: Objectives, Features and Technical Challenges", IEEE Communications Surveys \& Tutorials, Vol. 11(4), 2009, pp. 3-IS.

[20] Yusuf Perwej, Dr. Kashiful Haq, Uruj Jaleel , Sharad Saxena "Some drastic improvements found in the analysis of routing protocol for the Bluetooth technology using scatternet" Special Issue on The International Conference on Computing, Communications and Information Technology Applications (CCITA-2010), which is published by Ubiquitous Computing and Communication Journal (UBICC) Seoul, South Korea, Volume
CCITA-2010, No. 5 , pages 86-95, ISSN Online 1992-8424, ISSN Print 1994-4608.

[21] Taiji Kondo, Tamio Saito, Makoto Yoshida, "Technology for wifi/ Bluetooth And wimax coexistence", FUJITSU sci. tech. J, vol. 46, no. 1, april 62009.

[22] Z. Wang, D. Tsonev, S. Videv, H. Haas, "On the Design of a Solar-Panel Receiver for Optical Wireless Communications With Simultaneous Energy Harvesting", IEEE Journal on Selected Areas in Communications, vol. 33, no. 8, pp. 1612-1623, Aug 2015.

[23] D. Tsonev, S. Videv, H. Haas, "Unlocking spectral efficiency in intensity modulation and direct detection systems", IEEE J. Sel. Areas Commun., vol. 33, no. 9, pp. 1758-1770, Sep. 2015.

[24] H. H. Lu et al., "Bidirectional fiber-wireless and fiber-VLLC transmission system based on an OEO-based BLS and a RSOA", Opt. Lett., vol. 41, no. 3, pp. 476-479, 2016. 